\title{
Heart-type fatty acid-binding protein for risk assessment of chronic thromboembolic pulmonary hypertension
}

\author{
M. Lankeitt, ${ }^{*}$, C. Dellas*,+, A. Panzenböck" ${ }^{*}$ N. Skoro-Sajer* , D. Bonderman\#, \\ M. Olschewski ${ }^{\mp}$, K. Schäfer*, M. Puls*, S. Konstantinides* and I.M. Lang ${ }^{\#}$
}

ABSTRACT: Heart-type fatty acid-binding protein (H-FABP) is a reliable marker of myocardial injury and was recently identified as a predictor of outcome in acute pulmonary embolism. The aim of the present study was to investigate the prognostic value of H-FABP in chronic thromboembolic pulmonary hypertension (CTEPH).

In total, 93 consecutive patients with CTEPH were studied. During long-term follow-up (median duration 1,260 days, interquartile range (IQR) 708-2,460 days), 46 (49\%) patients had an adverse outcome, defined as CTEPH-related death, lung transplantation or persistent pulmonary hypertension after pulmonary endarterectomy (PEA).

Baseline H-FABP levels in plasma ranged from 0.69-24.3 $\mathrm{ng} \cdot \mathrm{mL}^{-1}$ (median (IQR) 3.41 (2.284.86) $\left.\mathrm{ng} \cdot \mathrm{mL}^{-1}\right)$. Cox regression analysis revealed a hazard ratio of $1.10(95 \%$ confidence interval 1.04-1.18) for each increase of H-FABP by $1 \mathrm{ng} \cdot \mathrm{mL}^{-1}$, and continuous elevations of H-FABP emerged as an independent predictor of adverse outcome by multivariable analysis. PEA was performed in $\mathbf{5 2}$ patients and favourably affected the long-term outcome. Kaplan-Meier analysis revealed that patients with baseline H-FABP concentrations $>2.7 \mathrm{ng} \cdot \mathrm{mL}^{-1}$, the median value of the biomarker in the surgically treated population, had a lower probability of event-free survival after PEA.

Heart-type fatty acid-binding protein is a promising novel biomarker for risk stratification of patients with chronic thromboembolic pulmonary hypertension.

KEYWORDS: Biomarkers, fatty acid-binding protein, prognosis, pulmonary hypertension

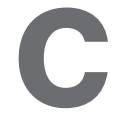

hronic thromboembolic pulmonary hypertension (CTEPH) is a life-threatening condition which is increasingly being recognised as one of the leading causes of severe pulmonary hypertension (PH) [1-3]. Its pathogenesis is still poorly understood and its association with venous thromboembolism remains controversial. This is partly because the factors predisposing to these two syndromes are not identical, and up to $60 \%$ of patients with CTEPH deny previous symptoms of acute pulmonary embolism (PE) $[4,5]$. Nevertheless, it is generally believed that single or recurrent thromboembolic events in the lung may initiate an aberrant process of thrombus organisation, followed by progressive pulmonary vascular remodelling and obliteration. Early studies suggested that only $0.1-0.5 \%$ of patients who survive an acute episode of $\mathrm{PE}$ eventually present with CTEPH [5], but more recent data indicate that the true proportion of patients developing this complication may be higher, ranging from $1-3.8 \%$ over a 2 - to $4-y r$ period $[6,7]$. Viewed in the context of an annual PE incidence of $\sim 50$ cases per 100,000 population [8], these rates highlight the disease burden imposed by CTEPH on patients and healthcare systems.

As in other forms of acute and chronic $\mathrm{PH}$, the natural course and prognosis of CTEPH are largely determined by the progressive development of right ventricular dysfunction and failure. Baseline clinical and haemodynamic findings are of established value in severity assessment and identification of candidates for pulmonary endarterectomy (PEA) [1]. In addition, a number of studies on pulmonary arterial hypertension, three of which included a small subgroup of patients with CTEPH (five to 19 individuals) [9-11], suggested that cardiac biomarkers such as troponin $\mathrm{T}$ and $\mathrm{N}$-terminal pro brain natriuretic

\section{AFFILIATIONS}

*Dept of Cardiology and Pulmonology, University of Goettingen, Goettingen, "Dept of Medical Biometry and Statistics, University Hospital of Freiburg, Freiburg, Germany, ${ }^{\#}$ Dept of Internal Medicine II, Division of Cardiology, Medical University of Vienna, Austria. +Both authors contributed equally.

\section{CORRESPONDENCE}

S. Konstantinides

Dept of Cardiology and Pulmonology University of Goettingen D-37099 Goettingen Germany Fax: 495513914131 E-mail: skonstan@med.unigoettingen.de

Received:

August 032007

Accepted after revision:

January 152008

SUPPORT STATEMENT

I.M. Lang was supported by grant No. S9406-B11 from the Fonds zur Förderung der wissenschaftlichen Forschung (FWF), Vienna, Austria.

STATEMENT OF INTEREST

None declared. 
peptide (NT-proBNP) might be helpful tools for optimising risk stratification.

Heart-type fatty acid-binding protein (H-FABP) appears to offer a number of theoretical and practical advantages over cardiac troponins for the detection of myocardial injury [12, 13]. Recently, Puls et al. [14] and KACZYÑSKA et al. [15] identified H-FABP as an early, highly sensitive and specific indicator of death or serious complications in acute PE. In the present study, the prognostic value of this biomarker was assessed in a large, single-centre population of 93 consecutive patients with CTEPH followed over a median period of $\sim 3.5$ yrs. The results indicate that H-FABP is more sensitive than cardiac troponins in predicting an adverse outcome in this setting. Elevated H-FABP levels at diagnosis may help identify patients with a high risk of death, a need for lung transplantation or persistent $\mathrm{PH}$ after PEA, and their prognostic value appears to be independent of baseline haemodynamic parameters.

\section{METHODS}

\section{Patient population and study design}

Between September 1994 and November 2005, 93 consecutive patients were diagnosed with $\mathrm{CTEPH}$, based on standardised criteria which included a mean pulmonary artery pressure $\left(\bar{P}_{\mathrm{pa}}\right)$ $>25 \mathrm{mmHg}$ at rest or $>30 \mathrm{mmHg}$ during exercise and a pulmonary vascular resistance (PVR) $>240 \mathrm{dyn} \cdot \mathrm{s} \cdot \mathrm{cm}^{-5}[16]$. $\mathrm{CTEPH}$ was confirmed as the cause of pulmonary arterial hypertension using established diagnostic protocols and imaging techniques, including ventilation-perfusion lung scanning, computed tomographic angiography and selective pulmonary angiography $[1,17]$. Haemodynamic parameters were determined by right heart catheterisation in all cases. Cardiac index and PVR were calculated based on Fick measurements of pulmonary flow. Complete baseline evaluation was performed at the Medical University of Vienna (Vienna, Austria), which serves as a primary referral centre for patients in Austria with suspected $\mathrm{PH}$ and particularly CTEPH.
Treatment consisted of oral anticoagulants, aimed at maintaining an international normalised ratio of 2.0-3.0, as well as diuretics, digitalis and oxygen at the physician's discretion. In addition, 52 patients ( $56 \%$ of the study population) underwent surgical PEA at various times during the follow-up period. The indications for PEA at the Medical University of Vienna have been described previously [18]. Briefly, patients were referred for PEA if they had a resting PVR $>300 \mathrm{dyn} \cdot \mathrm{s} \cdot \mathrm{cm}^{-5}$ (except for those with unilateral disease) and surgical accessibility of thromboembolic lesions. Surgery was not performed if PVR exceeded $1,100 \mathrm{dyn} \cdot \mathrm{s} \cdot \mathrm{cm}^{-5}$ or if the patient had severe comorbidity or refused surgical treatment [18].

An adverse outcome was defined as at least one of the following events during the follow-up period: 1) CTEPHrelated death; 2) need for lung transplantation; 3) persistent $\mathrm{PH}$ (as defined previously) despite PEA.

\section{Biomarker testing}

Venous plasma samples were collected at the time of initial diagnostic evaluation and at various times during the followup period. Samples were immediately stored at $-80^{\circ} \mathrm{C}$ and later analysed in batches after a single thaw.

Plasma levels of H-FABP (dilution 1:5) were measured by a solid-phase sandwich ELISA (HyCult Biotechnology, Uden, the Netherlands) as described previously [14]. The calibrators of the assay covered the range $0-25 \mathrm{ng} \cdot \mathrm{mL}^{-1}$. The minimum detection level was $0.25 \mathrm{ng} \cdot \mathrm{mL}^{-1}$ and the assay was linear within the range 0.1-25 ng. $\mathrm{mL}^{-1}$. Cardiac troponin $\mathrm{T}$ levels were determined using a quantitative electrochemiluminescence immunoassay (Elecsys 1010/2010 analyser; Roche Diagnostics, Mannheim, Germany) with a minimal detection limit of $0.01 \mathrm{ng} \cdot \mathrm{mL}^{-1}$.

The investigator who determined the biomarker levels was unaware of the patients' baseline parameters or clinical course. Biomarker levels were not used to guide patient management or monitor the effects of treatment during the initial hospital stay or at any time during the follow-up period. The study

TABLE 1 Baseline characteristics of 93 patients with chronic thromboembolic pulmonary hypertension (CTEPH)

\begin{tabular}{|c|c|c|c|c|}
\hline & Study patients & Adverse outcome ${ }^{\#}$ & No adverse outcome & $p$-value \\
\hline Subjects $(M / F) n$ & $93(47 / 46)$ & $46(25 / 21)$ & $47(22 / 25)$ & 0.536 \\
\hline 6MWD m & 317 (220-396) & $264(196-349)^{\bullet}$ & $356(237-401)^{+}$ & 0.027 \\
\hline NYHA functional class I-II/III-IV n & $16 / 69$ & $6 / 36^{\S}$ & $11 / 32^{f}$ & 0.279 \\
\hline PEA n (\%) & $52(56)$ & $22(48)$ & $30(64)$ & 0.146 \\
\hline mRAP mmHg & $10(6-14)$ & $11(8-17)$ & $9(5-11)^{\# \#}$ & 0.024 \\
\hline PCWP mmHg & $11(8-16)$ & $12(9-16)$ & $10(7-14)^{\# \#}$ & 0.067 \\
\hline $\mathrm{CO} \mathrm{L} \cdot \mathrm{min}^{-1}$ & $4.02(3.22-5.20)$ & $3.82(3.03-4.60)$ & $4.62(3.46-5.34)$ & 0.029 \\
\hline PVR dyn.s. $\mathrm{cm}^{-5}$ & $763(491-995)$ & $812(572-1125)$ & $700(447-848)$ & 0.023 \\
\hline
\end{tabular}

Data are presented as median (interquartile range), unless otherwise stated. M: male; F: female; 6MWD: 6-min walking distance; NYHA: New York Heart Association PEA: pulmonary endarterectomy; $\bar{P}$ pa: mean pulmonary artery pressure; mRAP: mean right atrial pressure; PCWP: pulmonary capillary wedge pressure; CO: cardiac output; PVR: pulmonary vascular resistance. ${ }^{\#}$ : adverse outcome was defined as CTEPH-related death, need for lung transplantation or persistent pulmonary hypertension after PEA; ${ }^{\circ}: n=20 ;{ }^{+}: n=26 ;{ }^{\text {s. }} n=42 ;{ }^{f}: n=43 ;{ }^{\# \#}: n=46$ 


\begin{tabular}{|c|c|c|c|c|}
\hline \multirow[t]{3}{*}{ TABLE 2} & \multicolumn{4}{|c|}{$\begin{array}{l}\text { Baseline heart-type fatty acid-binding protein }(\mathrm{H} \text { - } \\
\text { FABP) plasma levels and haemodynamic } \\
\text { parameters as predictors of adverse outcome in } \\
\text { chronic thromboembolic pulmonary } \\
\text { hypertension }\end{array}$} \\
\hline & \multicolumn{2}{|c|}{ Univariable model } & \multicolumn{2}{|c|}{ Multivariable model ${ }^{\#}$} \\
\hline & HR $(95 \% \mathrm{Cl})^{\circ}$ & $p$-value & HR $(95 \% \mathrm{Cl})^{\circ}$ & p-value \\
\hline $\mathrm{H}-\mathrm{FABP}^{+} \mathrm{ng} \cdot \mathrm{mL}^{-1}$ & $1.10(1.04-1.18)$ & 0.002 & $1.11(1.02-1.22)$ & 0.015 \\
\hline$\overline{P_{p a}}{ }^{\S} \mathrm{mmHg}$ & $1.03(1.01-1.06)$ & 0.010 & $1.00(0.95-1.05)$ & 0.943 \\
\hline $\mathrm{mRAP}^{\S} \mathrm{mmHg}$ & $1.10(1.05-1.16)$ & $<0.001$ & $1.07(1.01-1.13)$ & 0.025 \\
\hline $\mathrm{PCWP}^{\S} \mathrm{mmHg}$ & $1.07(1.02-1.12)$ & 0.005 & $1.023(0.96-1.09)$ & 0.530 \\
\hline $\mathrm{CO}^{f} \mathbf{L} \cdot \mathrm{min}^{-1}$ & $0.69(0.52-0.91)$ & 0.008 & $0.72(0.39-1.31)$ & 0.278 \\
\hline $\mathrm{PVR}^{\# \#}$ dyn.s.cm- & $1.00(1.00-1.00)$ & 0.010 & $1.00(1.00-1.00)$ & 0.199 \\
\hline PEA & $0.37(0.20-0.71)$ & 0.002 & $0.45(0.23-0.89)$ & 0.021 \\
\hline \multicolumn{5}{|c|}{ 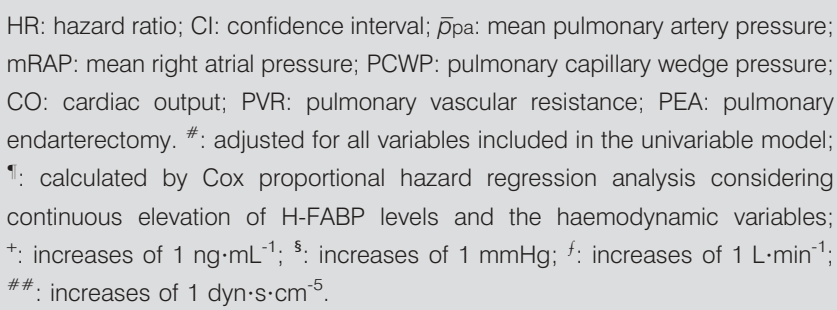 } \\
\hline
\end{tabular}

protocol was approved by the Ethics Committee of the Medical University of Vienna, and all patients gave informed consent.

\section{Statistical analysis}

The modified Kolmogorov-Smirnov test (Lilliefors test) was used to test for normal distribution of continuous variables. Continuous variables are expressed as medians with the corresponding interquartile range (IQR), and were compared using the Mann-Whitney U-test. Categorical variables were compared using Fisher's exact test. Spearman rank correlation was used to identify baseline variables related to the levels of $\mathrm{H}-\mathrm{FABP}$. The prognostic relevance of $\mathrm{H}-\mathrm{FABP}$ and other baseline parameters with respect to adverse long-term outcome, as defined previously, was estimated using Cox's proportional hazards model, which considered continuous elevation of H-FABP levels and the haemodynamic variables. The results are presented as hazard ratios (HR) with corresponding 95\% confidence intervals (CI), and were compared by Wald's test. The calculated HR and 95\% CI values refer to: $\mathrm{H}-\mathrm{FABP}$ increases of $1 \mathrm{ng} \cdot \mathrm{mL}^{-1} ; \bar{P}_{\mathrm{pa}}$, mean right atrial pressure (mRAP) and pulmonary capillary wedge pressure (PCWP) increases of $1 \mathrm{mmHg}$; cardiac output (CO) increases of $1 \mathrm{~L} \cdot \mathrm{min}^{-1}$; and PVR increases of $1 \mathrm{dyn} \cdot \mathrm{s} \cdot \mathrm{cm}^{-5}$. For the multivariable analysis, H-FABP and the univariable significant variables were entered into the Cox model simultaneously. Of note, cardiac troponin $\mathrm{T}$ was not considered in this model, since only four of the study patients had detectable levels at the time of diagnosis. In addition, eventfree survival rates for the type of treatment (PEA versus conservative) and for tertiles of H-FABP were estimated by the method of Kaplan-Meier and compared with the log-rank test. All tests were two-sided and used a significance level of 0.05 .

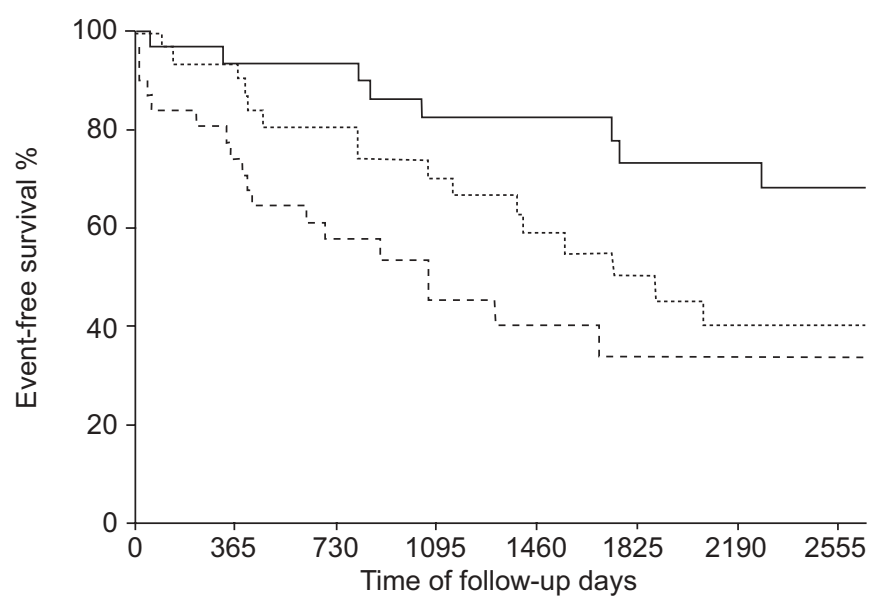

FIGURE 1. Elevated heart-type fatty acid-binding protein (H-FABP) levels were associated with a higher likelihood of an adverse outcome in patients with chronic thromboembolic pulmonary hypertension. Kaplan-Meier curves showing probability of event-free survival according to the tertiles of $\mathrm{H}$-FABP levels at initial diagnostic evaluation. ——: H-FABP <2.7 ng. $\mathrm{mL}^{-1} ; \cdots \cdots \cdot \mathrm{H}-\mathrm{FABP} 2.7-4.4 \mathrm{ng} \cdot \mathrm{mL}^{-1}$; ------: $\mathrm{H}$ $\mathrm{FABP}>4.4 \mathrm{ng} \cdot \mathrm{mL}^{-1}$. Difference between the lowest and the middle tertile, $\mathrm{p}=0.031$ (log-rank); difference between the middle and the highest tertile, $p=0.004$.

\section{RESULTS}

\section{Clinical and haemodynamic predictors of an adverse outcome of CTEPH}

The baseline clinical and haemodynamic findings of the study patients are shown in table 1. During the follow-up period, which ranged between 8 days and 13 yrs (median (IQR) 1,260 (708-2,460) days), 46 (49\%) patients had an adverse outcome. Of these, 33 patients died of CTEPH-related causes, five patients underwent lung transplantation (two of whom died of CTEPH-related causes during follow-up), and eight patients had persistent $\mathrm{PH}$ despite surgical treatment (PEA). In agreement with previous reports $[19,20]$, patients with an adverse outcome had a significantly shorter 6-min walking distance at baseline. They also had higher $\bar{P}$ pa, mRAP and PVR, and a lower $\mathrm{CO}$ at initial right heart catheterisation (table 1). Accordingly, these haemodynamic variables were associated with an increased risk of an adverse long-term outcome by univariable Cox regression analysis (table 2).

\section{Prognostic relevance of H-FABP concentrations at diagnosis}

At the time of initial diagnostic evaluation, H-FABP levels in plasma ranged from $0.69-24.3 \mathrm{ng} \cdot \mathrm{mL}^{-1}$ (median (IQR) 3.41 (2.284.86) $\left.\mathrm{ng} \cdot \mathrm{mL}^{-1}\right)$, and were weakly correlated with CO $(\mathrm{r}=-0.21$, $p=0.045)$, mRAP $(r=0.21, p=0.044)$, PCWP $(r=0.29, p=0.006)$ and the 6 -min walking distance $(r=-0.43, p=0.003)$. Baseline $\mathrm{H}-$ FABP concentrations were significantly higher in patients with an adverse outcome during follow-up compared with those with a favourable course (3.64 (2.86-5.37) versus 2.96 (2.164.49) $\mathrm{ng} \cdot \mathrm{mL}^{-1}$, respectively; $\left.\mathrm{p}=0.028\right)$. Univariable Cox regression analysis revealed an $\mathrm{HR}(95 \% \mathrm{CI})$ of 1.10 (1.04-1.18) for each increase of H-FABP by $1 \mathrm{ng} \cdot \mathrm{mL}^{-1} \quad(\mathrm{p}=0.002$; table 2$)$. Importantly, H-FABP emerged as an independent predictor of outcome by multivariable analysis that included baseline haemodynamic parameters and the type of treatment (table 2). Kaplan-Meier analysis (fig. 1) further supported the prognostic 


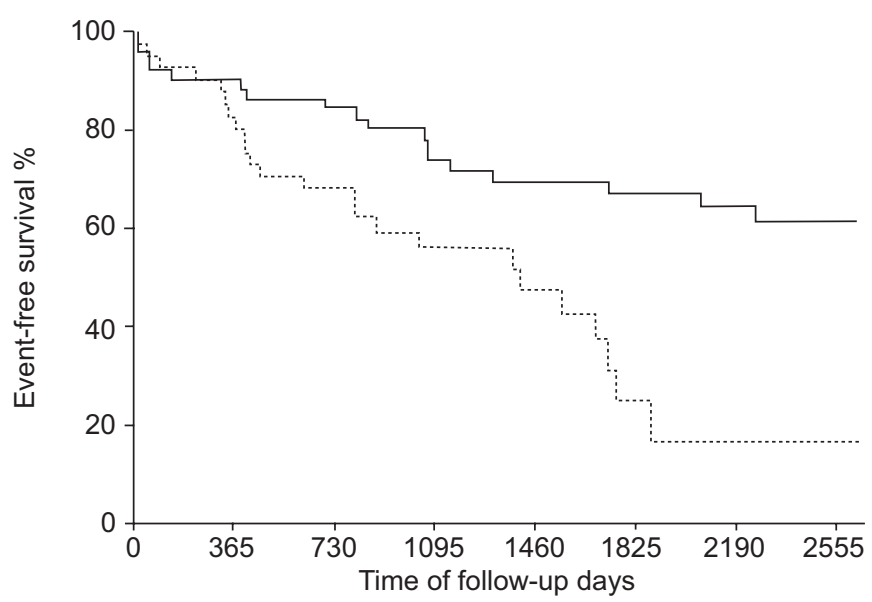

FIGURE 2. Kaplan-Meier curves showing probability of event-free survival for 52 patients who underwent pulmonary endarterectomy (PEA; - ) compared with 41 patients who received medical treatment (no PEA; …). $p=0.002$ by log-rank.

importance of the biomarker by showing that patients with $\mathrm{H}$ FABP levels in the lowest tertile $\left(<2.7 \mathrm{ng} \cdot \mathrm{mL}^{-1}\right)$ had a probability of event-free survival which was significantly higher than that of patients in the middle tertile $\left(2.7-4.4 \mathrm{ng} \cdot \mathrm{mL}^{-1} ; \mathrm{p}=0.031\right.$ by $\log$ rank test) or the highest tertile $\left(>4.4 \mathrm{ng} \cdot \mathrm{mL}^{-1} ; \mathrm{p}=0.004\right)$.

Surgical PEA has been reported to improve the prognosis of patients with CTEPH [21]. In the present study, 52 patients who underwent PEA had a significantly higher probability of eventfree survival during follow-up compared with those who were treated medically ( $\mathrm{p}=0.002$ by log-rank; fig. 2$)$. Univariable and multivariable analysis using the Cox model both confirmed that surgical treatment of CTEPH was associated with a significantly lower risk of an adverse outcome (table 2). Importantly, of the patients who underwent surgical treatment of CTEPH, those with an adverse long-term outcome had significantly higher baseline levels of H-FABP compared with those with a favourable outcome (median (IQR) $3.55(2.11-5.63) \mathrm{ng} \cdot \mathrm{mL}^{-1}$, $\mathrm{n}=22$, versus $2.29(1.68-3.49) \mathrm{ng} \cdot \mathrm{mL}^{-1}, \mathrm{n}=30$, respectively; $\mathrm{p}=0.026$ ). In agreement with this observation, Kaplan-Meier analysis showed a lower probability of event-free survival after PEA in patients with H-FABP concentrations $>2.7 \mathrm{ng} \cdot \mathrm{mL}^{-1}$ (the median value of this biomarker in the surgically treated population) at diagnosis ( $p=0.002$ by log-rank; fig. 3 ).

In contrast to a previous study in patients with pulmonary arterial hypertension of various aetiologies [11], only four $(4.3 \%)$ of the patients with CTEPH in the present study had detectable cardiac troponin $\mathrm{T}$ concentrations at diagnosis. All of these patients had an adverse outcome at follow-up.

\section{DISCUSSION}

Currently available biomarkers of myocardial injury, particularly the cardiac troponins I and T, are widely used in the triage of patients with acute coronary syndromes [22, 23]. Interestingly, cardiac troponin levels are also elevated in acute cor pulmonale resulting from PE [24], but it is unknown whether they can be used to detect right ventricular myocardial injury in the setting of chronic recurrent venous thromboembolic disease and CTEPH. Evidence has begun to accumulate that patients with pulmonary arterial hypertension

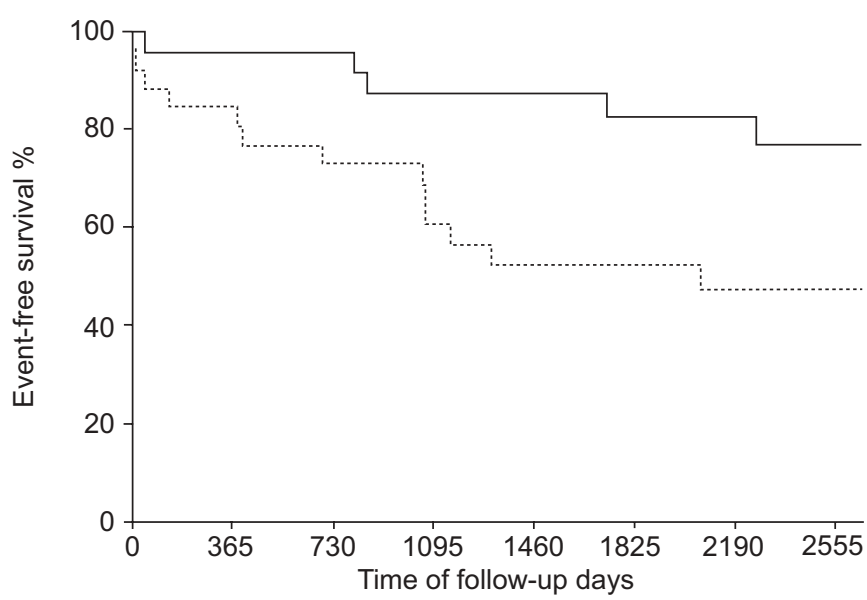

FIGURE 3. Elevated heart-type fatty acid-binding protein (H-FABP) levels were associated with a higher likelihood of an adverse outcome in patients with chronic thromboembolic pulmonary hypertension undergoing pulmonary endarterectomy. Kaplan-Meier curves showing probability of event-free survival in 52 operated patients according to dichotomised baseline H-FABP levels, i.e. below $(-)$ or above $(\cdots \cdots)$ the calculated median value in this population $\left(2.7 \mathrm{ng} \cdot \mathrm{mL}^{-1}\right)$; $\mathrm{p}=0.002$ by log-rank.

may exhibit prognostically relevant elevations of cardiac troponin or brain natriuretic peptide (BNP) levels in the circulation [9-11, 25, 26], but these studies included very few, if any, patients with CTEPH.

The present study focused on a population of 93 patients with CTEPH who were followed over a median period of $3.5 \mathrm{yrs}$; during this time, 52 (56\%) patients underwent surgical treatment (PEA) and 46 (49\%) patients had an adverse outcome defined as death of CTEPH-related causes, need for lung transplantation or persistent $\mathrm{PH}$ despite PEA. The present authors assessed the prognostic value of H-FABP, a novel, highly sensitive and specific biomarker of myocardial injury, which has yielded promising results in patients with acute coronary syndromes [12, 27] and, recently, in the setting of acute PE $[14,15]$. In the present study population with CTEPH, baseline H-FABP concentrations were significantly higher in patients with an adverse outcome during the follow-up period compared with those with a favourable course, and the probability of event-free survival progressively declined with increasing levels (tertiles) of $\mathrm{H}$ FABP in plasma. By Cox regression analysis, H-FABP levels, as well as mRAP and surgical treatment, emerged as independent predictors of an adverse outcome.

In contrast to $\mathrm{H}-\mathrm{FABP}$, the present authors found a disappointingly low prognostic sensitivity of cardiac troponin $\mathrm{T}$ in the patient population, as only four $(4 \%)$ patients had detectable troponin levels at baseline. All of these patients had an adverse outcome at follow-up; thus, cardiac troponin elevation appears, when present, to be an ominous prognostic indicator in CTEPH. Nevertheless, the present results partly contradict a previous report, which found this biomarker to be more frequently elevated in pulmonary arterial hypertension of various aetiologies [11]. Although the exact reasons for the apparent discrepancy are unclear, the earlier study included only a small number of patients with CTEPH. The findings of the present study indicate a superiority of H-FABP over 
troponin $\mathrm{T}$ in risk stratification of CTEPH and in the prediction of long-term event-free survival. These results are consistent with the recently reviewed theoretical advantages of H-FABP [12-14] including, for example, its small molecular size and favourable kinetics, its myocardial specificity which resembles that of the $\mathrm{MB}$ isoenzyme of creatine kinase, and its confinement to the cytoplasmic space.

The present study did not compare the prognostic value of $\mathrm{H}$ FABP to that of the natriuretic peptides BNP and NT-proBNP. Circulating levels of these latter biomarkers have been shown to correlate with the prognosis of patients with various forms of pulmonary arterial hypertension [9-11, 25, 26]. At present, the available evidence suggests that natriuretic peptides could be useful for monitoring the clinical course of patients with pulmonary arterial hypertension, and the effects of therapy [16], but the wide variations in the reported cut-off values for the assessment of cor pulmonale pose practical limitations to their routine use [28].

PEA is currently the treatment of choice for selected patients with CTEPH [29]. At present, the patient's functional status, together with invasively obtained haemodynamic parameters, is used to assess operability and guide therapeutic decisions [1,21,29]. In the present study, the independent favourable influence of PEA on the long-term outcome of patients with CTEPH was confirmed. Importantly, of the 52 patients who underwent surgical treatment of CTEPH, those with an adverse long-term outcome had significantly higher baseline levels of H-FABP compared with those with a favourable outcome. These findings suggest that $\mathrm{H}$ FABP measurements may help identify patients who could benefit most from surgical treatment. This clinically relevant hypothesis needs to be tested by larger, preferably prospective management, studies. In addition, further adequately powered studies are needed to determine whether serial measurements of H-FABP can be used as a surrogate marker for monitoring the response to surgical or medical therapy. In this regard, it also needs to be mentioned that the present study did not include patients treated with novel pharmacological agents, such as prostacyclin analogues, endothelin receptor antagonists and phosphodiesterase-5 inhibitors. Preliminary reports suggest that these drugs may favourably affect the clinical course of CTEPH [30].

In conclusion, the results of the present study indicate that heart-type fatty acid-binding protein is a reliable novel predictor of long-term outcome and, thus, a useful tool for risk stratification of patients with chronic thromboembolic pulmonary hypertension, including those undergoing pulmonary endarterectomy. The present findings provide the rationale for further studies to define the exact place of cardiac biomarkers and particularly heart-type fatty acid-binding protein, along with, or as an alternative to, haemodynamic parameters in management algorithms for this life-threatening disorder, which appears to affect more patients than previously thought.

\section{REFERENCES}

1 Hoeper MM, Mayer E, Simonneau G, Rubin LJ. Chronic thromboembolic pulmonary hypertension. Circulation 2006; 113: 2011-2020.

2 Lang IM. Chronic thromboembolic pulmonary hypertension-not so rare after all. N Engl J Med 2004; 350: 2236-2238.
3 Simonneau G, Galiè N, Rubin LJ, et al. Clinical classification of pulmonary hypertension. J Am Coll Cardiol 2004; 43: Suppl. 12, 5S-12S.

4 Lang I, Kerr K. Risk factors for chronic thromboembolic pulmonary hypertension. Proc Am Thorac Soc 2006; 3: 568-570.

5 Tapson VF, Humbert M. Incidence and prevalence of chronic thromboembolic pulmonary hypertension: from acute to chronic pulmonary embolism. Proc Am Thorac Soc 2006; 3: 564-567.

6 Becattini C, Agnelli G, Pesavento R, et al. Incidence of chronic thromboembolic pulmonary hypertension after a first episode of pulmonary embolism. Chest 2006; 130: 172 175.

7 Pengo V, Lensing AW, Prins MH, et al. Incidence of chronic thromboembolic pulmonary hypertension after pulmonary embolism. N Engl J Med 2004; 350: 2257-2264.

8 Stein PD, Kayali F, Olson RE. Regional differences in rates of diagnosis and mortality of pulmonary thromboembolism. Am J Cardiol 2004; 93: 1194-1197.

9 Andreassen AK, Wergeland R, Simonsen S, Geiran O, Guevara C, Ueland T. N-terminal pro-B-type natriuretic peptide as an indicator of disease severity in a heterogeneous group of patients with chronic precapillary pulmonary hypertension. Am J Cardiol 2006; 98: 525-529.

10 Fijalkowska A, Kurzyna M, Torbicki A, et al. Serum Nterminal brain natriuretic peptide as a prognostic parameter in patients with pulmonary hypertension. Chest 2006; 129: 1313-1321.

11 Torbicki A, Kurzyna M, Kuca P, et al. Detectable serum cardiac troponin $\mathrm{T}$ as a marker of poor prognosis among patients with chronic precapillary pulmonary hypertension. Circulation 2003; 108: 844-848.

12 Pelsers MM, Hermens WT, Glatz JF. Fatty acid-binding proteins as plasma markers of tissue injury. Clin Chim Acta 2005; 352: 15-35.

13 Alhadi HA, Fox KA. Do we need additional markers of myocyte necrosis: the potential value of heart fatty-acidbinding protein. QJM 2004; 97: 187-198.

14 Puls M, Dellas C, Lankeit M, et al. Heart-type fatty acidbinding protein permits early risk stratification of pulmonary embolism. Eur Heart J 2007; 28: 224-229.

15 Kaczyñska A, Pelsers MM, Bochowicz A, Kostrubiec M, Glatz JF, Pruszczyk P. Plasma heart-type fatty acid binding protein is superior to troponin and myoglobin for rapid risk stratification in acute pulmonary embolism. Clin Chim Acta 2006; 371: 117-123.

16 Galiè N, Torbicki A, Barst R, et al. Guidelines on diagnosis and treatment of pulmonary arterial hypertension. The Task Force on Diagnosis and Treatment of Pulmonary Arterial Hypertension of the European Society of Cardiology. Eur Heart J 2004; 25: 2243-2278.

17 Coulden R. State-of-the-art imaging techniques in chronic thromboembolic pulmonary hypertension. Proc Am Thorac Soc 2006; 3: 577-583.

18 Klepetko W, Mayer E, Sandoval J, et al. Interventional and surgical modalities of treatment for pulmonary arterial hypertension. J Am Coll Cardiol 2004; 43: Suppl. 12, 73S-80S.

19 Paciocco G, Martinez FJ, Bossone E, Pielsticker E, Gillespie B, Rubenfire M. Oxygen desaturation on the six-minute walk test and mortality in untreated primary pulmonary hypertension. Eur Respir J 2001; 17: 647-652. 
20 Miyamoto S, Nagaya N, Satoh T, et al. Clinical correlates and prognostic significance of six-minute walk test in patients with primary pulmonary hypertension. Comparison with cardiopulmonary exercise testing. Am J Respir Crit Care Med 2000; 161: 487-492.

21 Bonderman D, Skoro-Sajer N, Jakowitsch J, et al. Predictors of outcome in chronic thromboembolic pulmonary hypertension. Circulation 2007; 115: 2153-2158.

22 Korff S, Katus HA, Giannitsis E. Differential diagnosis of elevated troponins. Heart 2006; 92: 987-993.

23 Gibler WB, Cannon CP, Blomkalns AL, et al. Practical implementation of the Guidelines for Unstable Angina/ Non-ST-Segment Elevation Myocardial Infarction in the emergency department. Ann Emerg Med 2005; 46: 185-197.

24 Becattini C, Vedovati MC, Agnelli G. Prognostic value of troponins in acute pulmonary embolism: a meta-analysis. Circulation 2007; 116: 427-433.

25 Leuchte HH, Holzapfel M, Baumgartner RA, et al. Clinical significance of brain natriuretic peptide in primary pulmonary hypertension. J Am Coll Cardiol 2004; 43: 764-770.
26 Nagaya N, Nishikimi T, Uematsu M, et al. Plasma brain natriuretic peptide as a prognostic indicator in patients with primary pulmonary hypertension. Circulation 2000; 102: $865-870$.

27 Ishii J, Ozaki Y, Lu J, et al. Prognostic value of serum concentration of heart-type fatty acid-binding protein relative to cardiac troponin $\mathrm{T}$ on admission in the early hours of acute coronary syndrome. Clin Chem 2005; 51: 1397-1404.

28 Giannitsis E, Katus HA. Risk stratification in pulmonary embolism based on biomarkers and echocardiography. Circulation 2005; 112: 1520-1521.

29 Rubin LJ, Hoeper MM, Klepetko W, Galiè N, Lang IM, Simonneau G. Current and future management of chronic thromboembolic pulmonary hypertension: from diagnosis to treatment responses. Proc Am Thorac Soc 2006; 3: 601-607.

30 Bresser P, Pepke-Zaba J, Jaïs X, Humbert M, Hoeper MM. Medical therapies for chronic thromboembolic pulmonary hypertension: an evolving treatment paradigm. Proc Am Thorac Soc 2006; 3: 594-600. 